Conexiones naturales de la gran ciudad con las áreas periurbanas de Naturaleza.

Aplicación al caso de Madrid

Natural connections of the city with suburban natural areas. Application to Madrid

\title{
Alicia Bedmar Perlado :
}

Fecha de superación del Tribunal Fin de Máster: 14.07.2014

Tutor: José Fariña Tojo

\section{Resumen}

La movilidad peatonal se está convirtiendo cada vez más en un en objeto de atención para la sociedad. Después de un largo periodo de abandono debido al auge del transporte privado y al deterioro consecuente del espacio público, se mira al peatón como un agente regenerador de la ciudad, capaz de promover la integración social. Este hecho y la necesidad de acercar la Naturaleza a las ciudades motivan la búsqueda de soluciones para conseguir este fin.

Se probará una metodología basada en estudios recientes sobre movilidad peatonal, para posibilitar la llegada de los ciudadanos a las áreas de Naturaleza cercanas. Para probar y refinar la metodología se aplica a un estudio de caso, la ciudad de Madrid. El análisis de las posibles salidas de la ciudad, contrastará las conclusiones sobre la marcha a pie dentro de la ciudad. Como conclusión del estudio de caso se analizará la accesibilidad desde los distritos de Madrid a los puntos de Naturaleza cercanos.

\section{Palabras clave}

Movilidad sostenible, itinerarios peatonales, accesibilidad, servicios de ecosistemas

\section{Abstract}

The pedestrian mobility is becoming increasingly an object of attention for society. After a long time of promoting private transport, public spaces have deteriorated. Lately actions are directed to return the city to pedestrians and it is defined as an agent capable of promoting social integration. This fact and the need to bring Nature to cities motivate the search for solutions to achieve these finalities.

A methodology based on recent studies of pedestrian mobility will be tested to determine the possibility of access to nearby natural areas. To test and refine the methodology, it will be applied to a study case, Madrid City. The analysis of possible connections with nearby Nature will characterize the specific conditions of such routes, which will be different to the requirements of the pedestrian ways in the city. As a conclusion of the case study, we will analyze the accessibility from the districts of Madrid to the nearby natural areas.

\section{Keywords}

Sustainable mobility, pedestrian itineraries, availability, ecosystem services

- Alicia Bedmar Perlado es alumna de postgrado del Departamento de Urbanística y Ordenación del Territorio de la Escuela Técnica Superior de Arquitectura. Universidad Politécnica de Madrid, abedper@hotmail.com .

- Ref. Bib.: BEDMAR, A. (2015) "Conexiones naturales de la gran ciudad con las áreas periurbanas de Naturaleza. Aplicación al caso de Madrid", Territorios en Formación N.08, pp. XX-XX. 


\section{Introducción}

Durante los años sesenta y setenta, los espacios de la velocidad conquistan y transforman las ciudades (Mendoza, 2013). Triunfa el uso del vehículo privado por encima de cualquier otro. Así, paradas de transporte público, estaciones, lugares que habían sido emblemáticos en la ciudad por acercar el desarrollo, pierden su identidad y quedan convertidos en no-lugares, como explica Marc Augé (2007).

Como resultado nos encontramos con una ciudad fragmentada por una sucesión de vías que configuran un entramado difícil o imposible de sobrepasar, por lo que la ciudad ha quedado fragmentada en distintas celdas que generalmente funcionan de forma autónoma. La ciudad poco a poco ha ido perdiendo su condición de "paseable" ${ }^{1}$, cediendo cada vez más terreno al vehículo privado. Esta situación ha ocasionado múltiples problemas para el peatón, como consecuencia del tráfico que ha invadido las áreas entre edificios y dejando múltiples espacios residuales, "vacíos", en su interior (Comisión Europea, 2001). Aparecen los primeros síntomas de esta crisis: embotellamientos, alta accidentalidad, contaminación, tiempos de desplazamientos largos, ciudades dormitorio...

En estos últimos años, con una situación crítica por los problemas de insostenibilidad e incomodidad de las ciudades y motivados por los problemas ocasionados por el cambio climático, poco a poco van surgiendo planes y acciones, fundamentalmente al hilo de las teorías formuladas en la literatura europea y americana.

La circulación de las personas permite participar en la vida colectiva, de este modo el peatón se erige en elemento de integración social (Pozueta (dir.), Lamíquiz \& Porto, 2009). La tendencia en estos últimos años consiste en dar cada vez más protagonismo al peatón, devolverle su papel en la ciudad como el agente que da sentido al espacio urbano. Así la movilidad se ha convertido en un derecho, el "derecho a la movilidad".

Por otro lado la aspiración de mantenerse en contacto con una "acogedora y reparadora naturaleza" 2 es una aspiración recurrente a lo largo de la historia. A medida que la ciudad ha ido aumentando de tamaño, las áreas naturales se han ido alejando, por lo que se han buscado múltiples fórmulas para permitir este contacto. Ya en la historia moderna, desde la época industrial, el tema de acercar la naturaleza a las ciudades aparece de una forma sistematizada (desarrollo de ciudades jardín, greenbelts, etc.). En nuestros días, muchos autores, aunque en este caso nos refiramos a José Fariña, recuerdan la importancia de introducir naturaleza en las ciudades y la define como una infraestructura básica para el funcionamiento de la vida urbana.

\footnotetext{
1 Se utiliza la palabra "paseable" porque el trabajo La ciudad paseable de Pozueta, Lamíquiz y Porto (2009), entre otros documentos, han servido de esqueleto para organizar el contenido de una parte de este trabajo.

2 Hay que advertir que el origen de la fundación de las ciudades precisamente está en controlar el ambiente no habitado y poner separación física con la naturaleza. Como dice Fariña, los ciudadanos manipulaban el espacio para dar lugar a una organización distinta y mucho más controlada de las pequeñas porciones de territorio que abarcaba el recinto de la ciudad. Es decir, a estructuras urbanas. Esta manipulación no era, inicialmente, gratuita, sino que respondía a motivaciones más o menos conscientes. Para la sociedad urbana se trataba de convertir un espacio "natural (...), cuyo comportamiento le resultaba difícil de predecir, en un medio mucho más aprensible y que generara un espacio de mínima incertidumbre".
} 


\section{Objetivos}

Parece que hasta el final del siglo pasado y principios del presente las estrategias de movilidad llevadas a cabo por las distintas autoridades han seguido únicamente los dictados de las necesidades de movilidad motorizada. La articulación de la ciudad se ha identificado con una accesibilidad integral a todos los puntos del territorio para los vehículos a motor, llenando el territorio de distintas vías y carreteras que han dificultado la movilidad intrínseca y más natural del ser humano, la peatonal. Sin embargo, atendiendo a las conclusiones sobre los distintos estudios sobre cambio climático, en especial los dictados del Protocolo de Kioto y el objetivo de la Comisión Europea del "20-20-20", en estos últimos años empiezan a aparecer ciertas medidas y propuestas para favorecer una movilidad sostenible. ${ }^{3}$

No ocurre lo mismo con la necesidad de vertebrar la estructura urbana con las áreas naturales periféricas, donde la literatura al respecto es más escasa y el apoyo Institucional muy incipiente. Como ejemplos recientes, la Comisión Europea recoge una medida, la número 7, donde trata sobre el acceso a zonas ecológicas, aunque es en los planes locales donde más ejemplos existen en este sentido. Hay que advertir que este acceso "universal", perseguido en este trabajo, es a las áreas de naturaleza cercanas a la ciudad que, como se mostrará en el desarrollo del mismo, ya han sido antropizadas y han sido utilizadas por el hombre de distintas formas a lo largo de los años, bien como áreas ligadas a la agricultura, bien como cazaderos, objeto de romerías, etc.

Se plantea, pues, comprobar la viabilidad de acercar las áreas naturales a las ciudades de una manera universal, adecuada y eficaz, mediante la búsqueda de herramientas que permitan establecerlo. Para ello se elabora un método que, teniendo en cuenta las reflexiones existentes sobre movilidad peatonal y contrastando las características particulares de este tipo de recorridos, haga posible comprobar la viabilidad de acceder a estas áreas. Esta metodología, que en principio está basada en principios teóricos, será probada con un estudio de caso: se verificará la posibilidad de acceso universal -o natural- a las áreas de naturaleza periurbanas de Madrid.

\section{Hipótesis de partida}

Un entramado de vías e infraestructuras se extienden por todo el territorio, siendo en las ciudades y sus periferias donde esta trama se hace más densa. Así pues, la primera cuestión que se plantea es conocer si es posible encontrar una alternativa peatonal para conectar la ciudad con las áreas naturales circundantes.

Es necesario buscar las fórmulas que hagan posible esta conexión y verificar su aplicación en un estudio de caso. Así pues, la cuestión de partida es verificar si la aplicación de un método basado en la literatura existente sobre movilidad peatonal confirma esta hipótesis.

En segundo lugar, si se confirma esta cuestión, determinar si la trama urbana existente en las ciudades y su periferia se adapta a las necesidades de la movilidad peatonal y en concreto a esta

\footnotetext{
${ }^{3}$ Los Estados miembros se han comprometido a reducir para 2020 el consumo de energía primaria en un $20 \%$. No obstante, aún siguen existiendo numerosos obstáculos a la adopción de medidas eficaces para lograrlo. La Comunicación de la Comisión, de 13 de noviembre de 2008, denominada Eficiencia energética: alcanzar el objetivo del 20\% -COM(2008) 772- evalúa los proyectos futuros destinados a alcanzar los objetivos "20-20-20". En síntesis, el contenido de la Comunicación propone distintas medidas para mejorar la eficiencia energética, ya que esta contribuye a:

- la lucha contra el cambio climático,

- la mejora de la seguridad energética,

- la consecución de los objetivos de Lisboa,

- la reducción de costes en la Unión Europea.
} 
"movilidad peatonal de medio recorrido" que implica la salida de la ciudad para encontrarse con las áreas de naturaleza cercanas.

La tercera hipótesis que se plantea es determinar los requerimientos propios de este tipo de itinerarios y si difieren de los establecidos ya por varios autores para los recorridos peatonales urbanos.

\section{Metodología}

La cuestión de la conexión de las áreas naturales con la ciudad se aborda a través de la literatura recopilada sobre movilidad sostenible y en concreto la peatonal. Para ello se organizarán los conceptos fundamentales que intervienen en la definición de movilidad peatonal, así como los requisitos y condicionantes de ésta. Tres documentos han sido fundamentales para el desarrollo de este trabajo, a los que se ha incorporado nueva literatura y de autores clásicos (años sesenta y setenta). El hilo argumental ha sido tejido según los conceptos básicos y ejes de estos trabajos:

- La ciudad paseable. Recomendaciones para la consideración de los peatones, en el planeamiento, el diseño urbano y la arquitectura (Pozueta (dir.), Lamíquiz \& Porto, 2009).

- Implicaciones de la accesibilidad configuracional en la movilidad peatonal. El caso de Madrid (Lamíquiz, 2011).

- Pedestrians' Quality Needs (Comisión Europea, 2006).

El análisis y sistematización de los conceptos recogidos fundamentalmente en estos documentos, determinará los condicionantes y requerimientos necesarios para poder materializar la salida de las ciudades y llegar a las áreas naturales periurbanas.

Se verifica el método propuesto a través del estudio de caso en la ciudad de Madrid. Se analizarán los recorridos existentes en la ciudad y sus alrededores, para extraer datos necesarios para el análisis. La posibilidad de conexión se realiza a vista de pájaro sobre Madrid para verificar el curso de los recorridos.

\section{Marco de referencia}

Este trabajo analiza la conexión directa de la ciudad con las áreas naturales circundantes y en general con los "retazos de naturaleza" en la ciudad. Se define "conexión directa" a la que pueden establecer las personas por sus propios medios, es decir, a pie.

Se debe recalcar la importancia de la presencia de naturaleza en las ciudades. Tema ya recurrente en sucesivas publicaciones son los servicios de los ecosistemas que obtiene la sociedad, al conservar los espacios naturales donde habitan. Esta metodología adjudica un valor, muchas veces económico (sin duda discutible), a lo que representaría la pérdida de estos servicios.

\section{Conceptos principales}

Según Pozueta, Lamíquiz y Porto (2009), para que una ciudad sea paseable o, dicho con otras palabras, para que un entorno sea favorable al peatón, deben cumplirse estos cuatro requisitos fundamentales:

- Funcionalidad: Los equipamientos y servicios que se utilizan a diario deben estar ubicados en unos radios abarcables por el peatón, según unos tiempos determinados, influyendo de esta manera los impedimentos y barreras interpuestos. 
- Confortabilidad: Debe estar protegida por las inclemencias climáticas o de otra índole que hacen al peatón ser vulnerable.

- Seguridad: Debe ser segura tanto desde el punto de vista de la seguridad vial como de la seguridad ciudadana.

- Atractivo: Debe invitar a caminar, manteniendo interesado al peatón en su recorrido.

\section{Apoyo institucional a la movilidad peatonal}

Los temas principalmente tratados por los distintos agentes administrativos son los referentes a la movilidad sostenible. Este interés ha sido motivado por la preocupación creciente sobre el cambio del clima global y ha sido incluido en las estrategias para combatirlo; en concreto, la reducción de emisiones de efecto invernadero. Por tanto, se hace especial hincapié en el impulso de sistemas de transporte menos contaminantes, el fomento del transporte público y, de manera incipiente, la movilidad peatonal y ciclista.

Así, lo peatonal comienza a hacerse sitio en las distintas estrategias y planes europeos desde distintos enfoques como el medio ambiente, el transporte (en concreto, la movilidad sostenible) y la ordenación del territorio.

Los avances de las políticas europeas han sido recogidos en los principales documentos estratégicos del gobierno español y, en algunos casos, en leyes de movilidad, leyes del suelo y planes urbanísticos de distintas Comunidades Autónomas. Así, se incorporan conceptos como el urbanismo de proximidad, la oferta de transporte no motorizado, la permeabilidad de las infraestructuras para la fauna y los planes de movilidad sostenible. Estos últimos son responsabilidad de las administraciones autonómicas y locales. Muchas de ellas han empezado a desarrollar sus estrategias para una movilidad sostenible, siendo el caso de Vitoria, Andalucía, Madrid, Barcelona y Pamplona. Destaca el caso de Vitoria, que distingue la movilidad peatonal de la ciclista promoviendo sendos planes de movilidad.

Tanto en los planes autonómicos o locales, así como en el estatal, se resalta la necesidad de mejorar la seguridad vial del ciclista y del peatón y garantizar la intermodalidad de los medios no motorizados con los motorizados.

Por otro lado, existe un aporte interesantísimo por parte de los programas de investigación europeos, que brindan una nueva dimensión a los problemas de lo peatonal. Estudiarán las necesidades de calidad de los peatones que inciden en las decisiones de viaje y estancia de los peatones, sobre todo en las afueras de la ciudad (por el fenómeno del urban sprawl).

\section{Resultados: ¿Cómo salir de la ciudad a las áreas de Naturaleza periurbanas?}

\section{Definición de variables}

Se van a seguir las conclusiones de los trabajos realizados por la comisión de investigación europea COST en su trabajo Pedestrians' Quality Needs (Comisión Europea, 2006). Entre los factores involucrados en la marcha a pie se pueden distinguir varias etapas, que se van a sintetizar en el Cuadro 1.

Primero destacaremos la necesidad que motiva iniciar la marcha o el motivo del viaje. En relación al tema estudiado, se contemplan los siguientes motivos de viaje: el realizado por deporte, el realizado por ocio o salud y el realizado por motivaciones religiosas o culturales. 
Los condicionantes de la marcha a pie van a tener una decisión fundamental en las decisiones tácticas del recorrido:

- Accesibilidad (velocidad, distancia, tiempo recorrido): Lamíquiz (2011), en su tesis doctoral Implicaciones de la accesibilidad configuracional en la movilidad peatonal. El caso de Madrid, define la accesibilidad como «el conjunto de distancia, velocidad y tiempo de los recorridos».

- Vulnerabilidad / seguridad del peatón: seguridad vial y ciudadana. El peatón es el elemento más vulnerable de la movilidad, si se considera la marcha a pie como un medio de transporte. Se debe proteger al peatón del clima, del tráfico de los vehículos, de la accidentalidad o inseguridad ciudadana, de la contaminación, del ruido...

- Protección frente al clima: A lo largo de la historia urbana, la creación de microclimas artificiales más benignos para el transeúnte a base de pórticos, calles estrechas, soportales o incluso itinerarios subterráneos son recursos habituales en muchas ciudades. Sin embargo, este tipo de recursos se han ido abandonando tras el triunfo de la locomoción en automóvil y las corrientes estilistas de la arquitectura moderna.

- Topografía: Este condicionante es uno de los más determinantes en la marcha a pie. Sus consecuencias no se reducen al aumento del esfuerzos necesarios, sino que implican una reducción de la velocidad de desplazamiento y, por tanto, de la distancia potencial del radio de acción para la igualdad de duración del desplazamiento.

- Animación: Según Julio Pozueta (2009), los escenarios monótonos, sin interés visual y sin animación social pueden disuadir a los peatones mientras que, por el contrario, escenarios visualmente ricos y socialmente animados pueden animar a las personas a caminar. Así pues, en un entorno estimulante los itinerarios recorridos pueden ser mayores que en entornos desagradables. Es muy probable, continúa Pozueta, que modificando la percepción de los espacios a recorrer se incida en uno de los condicionantes del desplazamiento a pie, la distancia o el tiempo de recorrido.

- Atractivo visual: El atractivo de los espacios peatonales está muy condicionado por los usos de su entorno y el paisaje urbano. Es importante proponer el acondicionamiento de las vías para los peatones generando itinerarios más atractivos.

Los requisitos del entorno urbano van a modificar en cierta medida la percepción del recorrido, por lo que van a incidir directamente en la elección del trayecto. Los principales son los siguientes:

- Grado de dispersión urbana: La dispersión urbana (o urban sprawl) tiene unos efectos bien definidos sobre los desplazamientos. Primeramente, al separar las áreas urbanas entre sí aumentan las distancias entre origen y destino, lo que disuade al peatón para recorrerlos. Además, los espacios vacíos o residuales entre las distintas áreas urbanas actúan como barreras para los desplazamientos a pie, por la ausencia de sendas peatonales y actividad que hacen ser percibidos como inseguros.

- Densidad urbana. Afirma Julio Pozueta (2009) que a medida que las densidades urbanas disminuyen, las distancias medias interurbanas aumentan y a la inversa. En consecuencia, se puede suponer que al disminuir la densidad urbana se reducen las posibilidades de la marcha a pie. Buena parte del éxito de Madrid desde el punto de vista de la movilidad sostenible se debe a su modelo territorial. La fuerza y la compacidad de la ciudad central y la existencia de núcleos periféricos de densidades medias y altas favorecen el uso del transporte público (García \& Gutiérrez, 2005). 
- Mezcla de usos / disposición de los usos del suelo: Se debe destacar la capacidad de los barrios periféricos madrileños para implementar una movilidad con criterios de sostenibilidad. Son precisamente su alta densidad y diversidad de usos que han seguido al desarrollo urbanístico las que hacen posible que el transporte colectivo sea competitivo frente al uso del vehículo privado. No es así en el extrarradio, donde predominan las bajas densidades y un monocultivo residencial, fundamentalmente. En estas últimas es donde impera el transporte en vehículo privado hasta las nuevas ágoras o plazas urbanas, los centros comerciales (Lamíquiz, 2011).

- Trazado viario / red peatonal y su acondicionamiento: Un trazado viario bien acondicionado aumenta la accesibilidad de la red de espacios públicos.

- Diseño del edificio y calle pro-peatón / relación entre espacio público y la edificación: El paisaje urbano recorrido por los peatones se identifica en gran medida por los edificios. Condiciones como la separación del edificio respecto a la calle, la configuración y el grado de transparencia de las plantas bajas, las características intrínsecas de la edificación, el grado de apertura hacia la calle, los usos de las plantas bajas, etc. son rasgos que determinan la confortabilidad climática de la calle, la animación urbana, el interés del recorrido y la seguridad ciudadana.

\begin{tabular}{|c|l|l|}
\hline $\begin{array}{c}\text { Motivaciones del } \\
\text { viaje }\end{array}$ & \multicolumn{1}{|c|}{$\begin{array}{c}\text { decisiones tácticas } \\
\text { EL TRAYECTO } \\
\text { CONDICIONANTES DE LA MARCHA A PIE }\end{array}$} & $\begin{array}{c}\text { decisiones de uso } \\
\text { EL CAMINAR }\end{array}$ \\
\hline Ocio & Los RASGOS DEL ENTORNO
\end{tabular}

Cuadro 1. Síntesis de las etapas y factores que intervienen en los desplazamientos peatonales hasta las áreas naturales cercanas. (Fuente: Elaboración propia a partir de las conclusiones de varios autores).

\section{Método propuesto}

1. Caracterización del modelo periférico:

En primer lugar, se distingue la búsqueda de soluciones entre los modelos de crecimiento anglosajones y mediterráneos y entre los centros de las ciudades y las periferias, siendo estos temas importantísimos para la búsqueda de soluciones y el entendimiento de los problemas.

Así, en los modelos de crecimiento anglosajones, la expansión de la ciudad ha sido de baja densidad, haciendo de la utilización del vehículo privado la forma de transporte por excelencia. Sin embargo, en los modelos de crecimiento mediterráneos, este crecimiento se ha producido fundamentalmente en concentraciones de gran densidad, haciendo que el transporte público tenga mucha eficacia y sea competitivo. No obstante, en los últimos años, la expansión de las ciudades se ha generalizado asimilando el modelo anglosajón. Este modelo de crecimiento es el denominado urban sprawl, característico de las ciudades europeas contemporáneas.

La conexión de las ciudades con sus áreas naturales cercanas consta de varios tipos de itinerarios con características y condicionantes muy distintos entre sí. En primer lugar nos encontramos con unos itinerarios urbanos que deben cumplir los requisitos establecidos por los autores estudiados; en segundo lugar, unos itinerarios que discurren por ámbitos periféricos y, después, rurales. Esto constituye, en la mayor parte de los casos, itinerarios de medio-largo recorrido, que tendrán unos condicionantes sensiblemente diferentes a los puramente urbanos. 
Entre estas diferencias destacamos el propósito mismo del viaje. Ya hemos indicado que los propósitos del viaje se restringen a los deportivos, ocio, salud, ritos o costumbres.

\section{Definición de los usuarios:}

En este caso, las distancias a recorrer no serán las puramente funcionales del ámbito urbano, sino que van a depender más bien de la capacidad de resistencia del peatón, abarcando mayores distancias. Así pues se propone una clasificación en usuarios según su capacidad de abarcar distancias:

- Usuarios con necesidades especiales: este grupo lo constituyen niños, ancianos y ciertas personas con discapacidad. A este grupo se le asignan tiempos de recorrido menores a 1 hora.

- Usuario tipo: con una capacidad física media, capaz de caminar durante 1 ó 2 horas.

- Usuario entrenado: con una capacidad física mayor debida al entrenamiento, siendo capaces de caminar durante una jornada de aproximadamente 8 horas.

3. Caracterización de una red apropiada para el peatón:

Tanto en la ciudad como en el territorio en el que se desenvuelven, existen una multiplicidad de vías y posibles conexiones que, sorteando las barreras que se han ido interponiendo en aras del vehículo motorizado, conectan con las áreas naturales deseadas. De este entramado de posibles vías las más adecuadas serán las que reúnan las mejores características que condicionan la marcha a pie. De nuevo obtenemos una trama ya menos densa pero que va a hacer más confortable el viaje al peatón. Las condiciones que deben cumplir están expresadas en el Cuadro 2. En este cuadro, además, se relaciona la manera en la que las características del entorno inciden en los condicionantes en las distintas etapas del método propuesto (Cuadro 3).

Obtener una red posibilista para salir de cualquier punto de la ciudad tiene ventajas, seguramente asociadas a la libertad de movimiento por la ciudad, pero también desventajas, ligadas con la operatividad de esta red, resultando poco trazable, difícil de acondicionar y mantener y de difícil utilización del ciudadano, por no poder seguir un itinerario concreto.

De manera sintética, se enumeran los pasos descritos anteriormente.

Paso 1: Definición de la red de conexión o "red amable".

- Identificación de las áreas naturales, puntos de destino: Los puntos de acceso a las áreas naturales están ya generalmente resueltos, ya que estarán siendo utilizados y recogidos en guías y distintas publicaciones. En caso contrario se recogerá una muestra de puntos de acceso significativa, compatible con el uso y grado de protección del área natural.

- Identificación de los puntos de origen: Para determinar los puntos de partida en la ciudad se proponen puntos representativos, conocidos por los vecinos del barrio y ubicados en posiciones de centralidad. Se tomarán tantos puntos como sean representativos de la ciudad en cuestión.

- Definición de barreras: Es interesante conocer las barreras en el territorio, tanto naturales (ríos, fuertes pendientes, barrancos, etc.), como artificiales. Y dentro de estas últimas, barreras lineales (viarios para la velocidad, vallados y cercados, etc.) y 
puntuales (grandes infraestructuras). Estas serán determinantes para abordar el siguiente paso.

- $\quad$ Factores que intervienen en la marcha peatonal: Como ya se ha dicho, obtenemos una red con múltiples soluciones de conexión, una red de espacio público capaz de cumplir con las necesidades del peatón a la hora de iniciar la marcha a pie. Pero conexión no quiere decir accesibilidad y, por tanto, habrá caminos que no podrán ser utilizados. Así que, por último, será necesario valorar la accesibilidad desde distintos puntos de la ciudad a otros ubicados en las áreas de naturaleza, teniendo en cuenta factores como: el atractivo o la animación, la seguridad vial ciudadana, las condiciones del microclima y la topografía.

- Estudio de preexistencias: Ya que el contacto con la Naturaleza es una necesidad recurrente, existe un número limitado de rutas utilizadas por peatones y ciclistas, quienes, gracias a las nuevas tecnologías (asociadas al GPS), registran y posteriormente publican en distintos portales de internet. Otras rutas más tradicionales han sido publicadas por la Administración o recogidas en guías senderistas. En este sentido, las tradiciones y la cultura popular, ligadas a las tradicionales romerías, acontecimientos históricos y otros muchos eventos, nos han dejado sus rastros en el territorio, que en algunos casos son fáciles de rastrear. Otros elementos ligados a los usos tradicionales del suelo han dejado su particular huella, siendo a su vez apropiados para la marcha peatonal, como es el caso de las vías pecuarias.

Paso 2: Determinar la accesibilidad.

- Selección de itinerarios según el grado de accesibilidad de los recorridos: Una vez se han determinado los puntos de salida y llegada, se puede hablar de itinerarios y, por tanto, de la accesibilidad de éstos o, lo que es lo mismo, de la accesibilidad de la ciudad (o distintos puntos de la ciudad) a las áreas naturales circundantes (o puntos de éstas).

- Se establecen cuatro tipos de accesibilidad peatonal, correspondientes a los tipos de usuarios: básica, moderada, limitada y no accesible, según el siguiente cuadro.

\begin{tabular}{cccc}
\hline $\begin{array}{c}\text { DEFINICIÓN DE } \\
\text { ACCESIBILIDAD }\end{array}$ & USUARIO & DISTANCIA $(\mathrm{m})$ & TIEMPO (horas) \\
\hline BÁSICA & necesidades especiales & $0-5.000$ & $1 \mathrm{~h}$ aprox. \\
\hline MODERADA & medio & $5.000-10.000$ & de 1 a 2 horas \\
\hline LIMITADA & entrenado & $10.000-30.000$ & $8 \mathrm{~h}$ aprox. \\
\hline NO ACCESIBLE & - & $>30.000$ & $>8 \mathrm{~h}$ \\
\hline
\end{tabular}

Cuadro 2. Accesibilidad de los itinerarios peatonales que conectan con las áreas de Naturaleza periféricas. (Fuente: Elaboración propia a partir de datos publicados por Pozueta (2008) y Molina \& Sanz (1980)).

- Necesidades de mejora de los trazados en función de los condicionantes de la marcha a pie: Por último, sobre los itinerarios resultantes, se podrán determinar aquellos más interesantes, acondicionar, señalizar cualquier operación pertinente o establecer las necesidades de mejora de los trazados en función de los condicionantes de la marcha a pie. 


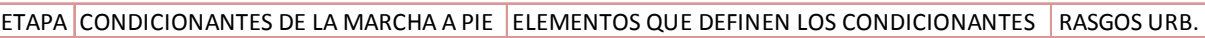

1.- DEFINICIÓN DE LA RED DE CONEXIÓN CON LAS ÁREAS DE NATURALEZA PERIURBANAS

\begin{tabular}{ll|l|}
\hline ATRACTIVO O ANIMACIÓN & animación social \\
\hline & usos del entorno \\
\hline SEGURIDAD VIAL y CIUDADANA & paisaje \\
\hline & tráfico de los vehículos \\
\hline & accidentalidad \\
\hline & inseguridad ciudadana \\
\hline CONDICIONES DEL MICROCLIMA & contaminación y ruido \\
\hline & animación: autovigilancia \\
\hline & relacionadas con la calle (pórticos, calles estrechas) \\
\hline & arbolado de alineación \\
\hline TOPOGRAFÍA & vegetación \\
\hline & presencia de humedad \\
\hline & pendientes máx. para bicicletas $<4 \%$ \\
\hline & pendientes máx. para peatón $<6 \%$ \\
\hline
\end{tabular}

2.- SELECCIÓN DE ITINERARIOS DESDE PTOS CONCRETOS DE LA RED A PTOS CONCRETOS DE LAS ÁREAS NATURALES

\begin{tabular}{ll}
\hline ACCESIBILIDAD & \\
\hline (velocidad+tiempo+distancia) & barreras lineales (viario de velocidad, infraestr., rios...) \\
& pendientes excesivas
\end{tabular}
pendientes excesivas desvíos (por el propio trazado de la vía)

1 DISEÑO VÍA Y RELAC. CON EDIFIC.

2 CONFIGURACIÓN RED PEATONAL

3 DISPOSICIÓN DE USOS

4 DENSIDAD

Cuadro 3. Relación con los condicionantes de la marcha a pie y los rasgos urbanos. (Fuente: Elaboración propia contrastando las conclusiones de Lamíquiz (2011) y Pozueta (2008) y el estudio de caso propuesto).

\section{Resultados: Aplicación del método}

Según los pasos descritos anteriormente, se ha probado la metodología en la ciudad de Madrid.

\section{Identificación del área de estudio}

- Selección de los puntos de destino: Debido al alto valor natural, histórico y cultural inherente en este territorio, existen varias figuras de protección que abarcan gran parte de la geografía madrileña. Formando parte de éstas, se estudia el uso que se les da en la actualidad, la accesibilidad de las rutas existentes y los puntos preferidos por los madrileños.

- Selección de los puntos de origen: Para trazar un itinerario es necesario establecer un punto de llegada y de salida. Para los puntos de salida es importante que sean puntos reconocibles en la ciudad, que tengan cierta entidad y representatividad y que se ubiquen en posiciones céntricas.

Se han considerado cinco sectores de Madrid, correspondientes a los cuatro puntos cardinales y al centro. A estos sectores se les ha asignado los distritos correspondientes. Para seleccionar los puntos de partida, en este caso, se han seleccionado las Juntas Municipales de Distrito, por ser edificios representativos, ampliamente conocidos y estar localizados en puntos del distrito con mayor actividad.

\section{Determinación de las posibles rutas}

Se ha realizado un rastreo de las posibles salidas naturales desde la ciudad de Madrid, concluyendo como las más probables las siguientes: 
- Salidas a las áreas de naturales en el pasado: Como se puede ver, el encuentro y disfrute de la Naturaleza ha estado vinculado desde tiempos remotos con la tradición, los usos y costumbres en forma de romerías, peregrinaciones o simplemente por combatir las inclemencias climáticas de la ciudad (los baños en el Manzanares y la Playa de Madrid). En estos casos, el fin último era el encuentro mismo con la Naturaleza y para ello se pusieron los medios necesarios para conectar las ciudades con estos espacios (el Puente de Segovia, por ejemplo, fue construido con este fin).

Varias causas han originado la pérdida de estos espacios. En el caso del Manzanares primero la contaminación del río y la posterior interposición de la M-304. En este punto es destacable la operación Río, que hace que vuelvan a ser practicables estos recorridos.

Otros todavía perduran, quedando como reductos de Naturaleza en la ciudad y siguen manteniendo sus costumbres, aunque obviamente el pequeño peregrinaje realizado entonces ya no es posible. Es el caso de la Pradera de San Isidro.

- Salidas de la ciudad en la actualidad: Los itinerarios utilizados hoy en día para moverse por Madrid y su periferia en bici o a pie están publicados en guías y rutas de senderismo $y$, de manera muy interesante, compartidos en internet por los ciclistas o peatones que van descubriendo rutas nuevas. Es un medio novedoso, actualizado constantemente, que deja gran número de datos disponibles. Se ha accedido a las cuatro fuentes más visitadas por internet, son páginas confeccionadas por asociaciones anónimas, pero también el Ayuntamiento de Madrid, a través de la Oficina de la bici, ha publicado las rutas e itinerarios más adecuados para moverse por Madrid y sus alrededores en bicicleta.

- Caminos tradicionales: Corresponden a los caminos históricos que parten de Madrid y al trazado de vías pecuarias.

- Caminos históricos: son rutas de largo recorrido que parten de Madrid. Se han localizado tres rutas que han sido recuperadas recientemente: la Senda Real (GR-124), el tramo madrileño del Camino de Santiago (GR-10) y la Senda de las Merinas.

- Red de vías pecuarias: Son las rutas o itinerarios por donde discurre o ha venido transcurriendo tradicionalmente el tránsito ganadero, para aprovechar los pastos en las dehesas de verano o de invierno. Son un legado histórico de interés capital, único en Europa.

Son rutas interesantes, ya que en general mantienen un trazado integral durante muchos kilómetros. El recorrido de estos caminos dependerá de la preparación física del peatón o de las etapas las que se quiera recorrer.

Prácticamente la totalidad de los itinerarios que conectan la ciudad con las áreas naturales cercanas están siendo usados con mayor o menor comodidad por peatones y ciclistas. En muchos casos, son los propios usuarios de estas vías los que señalizan en el camino de manera no ortodoxa a base de grafitis o diversos mojones los recorridos preferidos.

En estos itinerarios más recientes, el trazado de rutas gracias a las nuevas tecnologías que permiten la georreferenciación de éstas a través de dispositivos GPS y la rápida difusión en portales de internet genera la consolidación de itinerarios descritos por los usuarios.

${ }^{4}$ Es de destacar, que los baños en el Manzanares no han dejado nunca de existir, sino que se han trasladado aguas arriba, al Monte de El Pardo, donde no hay contaminación. 
Es fácil determinar entre las rutas difundidas el motivo del viaje. Se han recogido aquellas que lleguen a los puntos de destino deseados o enlacen con otra ruta que acceda y en los que el motivo del viaje sea el ocio, la salud o el deporte.

De otros caminos históricos o tradicionales todavía quedan testigos de su existencia pasada, como arbolado, retazos de caminos o restos de distintos pavimentos. En algunos casos su reutilización consiste en salvar las distintas barreras que se extienden por el territorio y, en otros, simplemente cortar ciertas alambradas que impiden el paso (Ecologistas en Acción, 2003).

Es importante resaltar el papel de las vías pecuarias, ya que se abren camino por el medio rural, también por el urbano, aunque en este último hayan sido cubiertas de asfalto, perdiendo así una de sus características determinantes como vías pecuarias. El acceso a las áreas naturales en muchos casos no es fácil, ya que un gran número de ellas han sido cortadas por vías de comunicación o ferrocarril y no se han construido pasos que aseguren su continuidad. No obstante, se han identificado aquellas o trozos de estas que enlacen con otros caminos y lleguen a los puntos de destino deseados.

\section{Cumplimiento de los condicionantes de la marcha a pie e influencia de las variables del entorno urbano}

Por otro lado, las condiciones del entorno van a modificar la percepción de estos recorridos, incidiendo en algunas de las variables expuestas anteriormente (Cuadro 4). Como ya se indicó en la metodología, al ser un análisis a vista de pájaro, los rasgos urbanos que influyen en la movilidad peatonal se han generalizado para la distinción de tramos expuesta (Cuadro 5).

En una ciudad de la escala de Madrid, las características del entorno varían siguiendo el modelo de crecimiento que ha seguido la ciudad. Se reconocen tres entornos y sus características principales:

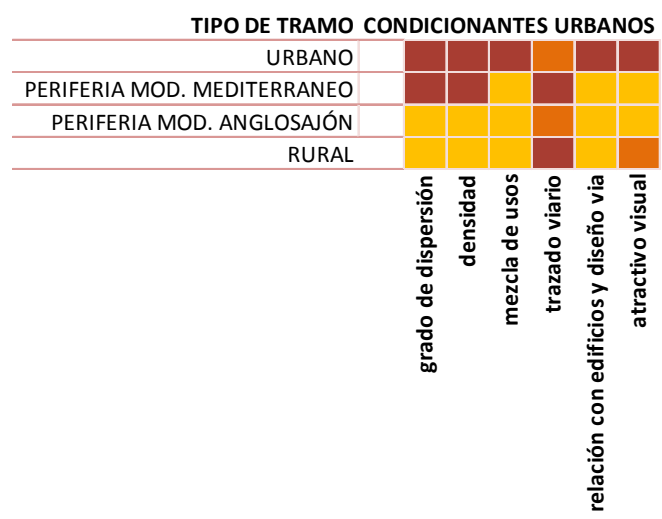

Cuadro 4. Relación de los distintos tramos de los itinerarios de conexión con los condicionantes urbanos. (Fuente: Elaboración propia a partir de las conclusiones de varios autores).

- Entorno urbano: Denso, poco disperso, con gran variedad de usos y alta posibilidad de encontrar atractivo. El trazado viario es el resultante de la ocupación del suelo y la posterior adaptación al movimiento a motor, lo que causa que muchas veces, los trazados no tengan demasiadas alternativas de diseño (trazado, dimensiones de la sección, etc.). 
- Entorno periférico: Agrupaciones de ciudad dormitorio, con densidades altas y moderadas $^{5}$, poca diversidad de usos, en general menor relación del viario con la edificación y mayor presencia del vehículo privado que invade parte del espacio público.

- Entorno rural: El que todavía puede encontrarse previamente al ingreso a las áreas naturales. A menudo despreciado por la ciudad próxima que se desarrolla a espaldas de este espacio. En ocasiones, esto origina que se encuentre degradado, incluso con presencia de vertidos ilegales y usos degradantes. Presenta escasa vegetación (insuficiente resguardo del clima). Poco denso y disperso, ocupado tradicionalmente por tierras de labor, hoy en día abandonadas. La ventaja es que hay espacio suficiente para dimensionar y trazar itinerarios adecuados. El paso por estos terrenos supondría la puesta en valor de los mismos, hecho que podría suponer una mejora de estos paisajes.

\begin{tabular}{|c|c|c|c|}
\hline \multirow[t]{5}{*}{$\begin{array}{l}\text { entorno } \\
\text { urbano }\end{array}$} & topografía & $\begin{array}{l}<4 \% \text { bicis } \\
<6 \% \text { peaton }\end{array}$ & Se toman pendientes del $4 \%$ por ser más restrictivas \\
\hline & seguridad vial & $\begin{array}{l}\text { calles tranquilas } \\
\text { anchos aceras suficientes }\end{array}$ & $\begin{array}{l}\text { sin tráfico excesivo y recomendadas por el Ayuntamiento de Madrid } \\
\text { debido a la extensión del ámbito no se ha tenido en cuenta }\end{array}$ \\
\hline & seguridad ciudadana & calles animadas & debido a la extensión del ámbito no se ha tenido en cuenta \\
\hline & microclima & $\begin{array}{l}\text { calles estrechas } \\
\text { calles arboladas } \\
\text { pasos por parques y jardines }\end{array}$ & $\begin{array}{l}\text { debido a la extensión del ámbito no se ha tenido en cuenta } \\
\text { no se han encontrado datos } \\
\text { se han pasado por estos puntos según posibilidad del recorrido }\end{array}$ \\
\hline & escena urbana & $\begin{array}{l}\text { calles animadas } \\
\text { recorrido por hitos } \\
\text { pasos por parques y jardines }\end{array}$ & $\begin{array}{l}\text { debido a la extensión del ámbito no se ha tenido en cuenta } \\
\text { debido a la extensión del ámbito no se ha tenido en cuenta } \\
\text { se han pasado por estos puntos según posibilidad del recorrido }\end{array}$ \\
\hline \multirow[t]{5}{*}{ periferia } & topografía & $\begin{array}{l}<4 \% \text { bicis } \\
<6 \% \text { peaton }\end{array}$ & no se han encontrado datos \\
\hline & seguridad vial & $\begin{array}{l}\text { calles tranquilas } \\
\text { anchos aceras suficientes } \\
\text { itinerarios utilizados }\end{array}$ & $\begin{array}{l}\text { sin tráfico excesivo y recomendadas por el Ayuntamiento de Madrid } \\
\text { debido a la extensión del ámbito no se ha tenido en cuenta } \\
\text { distintas fuentes }\end{array}$ \\
\hline & seguridad ciudadana & $\begin{array}{l}\text { calles animadas } \\
\text { itinerarios utilizados }\end{array}$ & $\begin{array}{l}\text { debido a la extensión del ámbito no se ha tenido en cuenta } \\
\text { distintas fuentes }\end{array}$ \\
\hline & microclima & $\begin{array}{l}\text { calles arboladas } \\
\text { pasos por parques y jardines }\end{array}$ & $\begin{array}{l}\text { no se han encontrado datos } \\
\text { se han pasado por estos puntos según posibilidad del recorrido }\end{array}$ \\
\hline & escena urbana & $\begin{array}{l}\text { calles animadas } \\
\text { recorrido por hitos } \\
\text { pasos por parques y jardines } \\
\text { evitar zonas industriales }\end{array}$ & $\begin{array}{l}\text { debido a la extensión del ámbito no se ha tenido en cuenta } \\
\text { debido a la extensión del ámbito no se ha tenido en cuenta } \\
\text { se han pasado por estos puntos según posibilidad del recorrido } \\
\text { se han evitado según posibilidad del recorrido }\end{array}$ \\
\hline \multirow[t]{5}{*}{ rural } & topografía & $\begin{array}{l}<4 \% \text { bicis } \\
<6 \% \text { peaton }\end{array}$ & no se han encontrado datos \\
\hline & seguridad vial & $\begin{array}{l}\text { pasos e intersecciones } \\
\text { seguras con vías motorizadas }\end{array}$ & se han evitado siempre las intersecciones inseguras \\
\hline & seguridad ciudadana & itinerarios utilizados & no siempre conectan con las áreas deseadas \\
\hline & microclima & - & difícil encontrar elementos de protección \\
\hline & atractivo del paisaje & $\begin{array}{l}\text { áreas con vistas o atractivas } \\
\text { evitar usos degradantes }\end{array}$ & $\begin{array}{l}\text { al ser un análisis a vista de pájaro es difícil comprobar este punto } \\
\text { se han evitado según posibilidad del recorrido }\end{array}$ \\
\hline
\end{tabular}

Cuadro 5. Condicionantes para la movilidad peatonal en el caso de estudio. (Fuente: Elaboración propia a partir de las conclusiones de varios autores y el análisis del caso de estudio).

\footnotetext{
5 Según García y Gutiérrez (2005), en el caso del municipio de Madrid, la periferia corresponde a un modelo de crecimiento mediterráneo, caracterizado por agrupaciones de alta densidad y unos usos muy homogéneos que dan lugar a las denominadas ciudades dormitorio.
} 


\section{Accesibilidad a las áreas de naturaleza periurbanas}

- Detección de barreras: Las consecuencias del modelo de movilidad planteado en la actualidad plantea un gran número de externalidades, a saber, tráfico intenso en determinados puntos, accidentalidad, contaminación atmosférica, ruido, etc. Así pues, las barrearas interpuestas inciden directamente en varios de los condicionantes de la marcha a pie, la seguridad vial, la accesibilidad por prolongar los recorridos y, en menor medida, el atractivo del paisaje.

- Estudio sobre la accesibilidad de las posibilidades de conexión: Obtener una red posibilista para salir de cualquier punto de la ciudad tiene ventajas, seguramente asociadas a la libertad de movimiento por la ciudad, pero también desventajas, ligadas con la operatividad de esta red, resultando poco trazable, difícil de acondicionar y mantener y de difícil utilización por el ciudadano, ya que moverse por una red implica cierta desorientación.

Esta red, que se puede denominar "red amable para el peatón", cuenta con múltiples soluciones de conexión. En definitiva, es una red de espacio público capaz de cumplir con las necesidades del peatón a la hora de iniciar la marcha a pie. Pero conexión no quiere decir accesibilidad y, por tanto, habrá caminos que no podrán ser utilizados, ya que se propone llegar a las áreas de naturaleza alcanzables en una jornada. Así que, por último, será necesario valorar la accesibilidad desde distintos puntos de la ciudad a otros ubicados en las áreas de Naturaleza.

Como vimos en el capítulo de áreas de Naturaleza y caminos existentes, se han valorado las rutas efectuadas por los madrileños en tiempo, distancia, velocidad media y dificultad de la ruta, llegando a las siguientes conclusiones:

Es interesante ver cómo, para los datos de la muestra recogidos, la bicicleta recorre una distancia sensiblemente menor que el peatón entrenado, claro que los recorre en mucho menos tiempo. Esto demuestra que el dato obtenido para la accesibilidad en términos de distancia es el mismo para los dos tipos de usuario, el ciclista y el peatón, y que el techo de kilómetro para este tipo de recorridos ronda los 25 kilómetros. Teniendo en cuenta que una persona en buena forma física y entrenada puede caminar alrededor de 8 horas, lo que supone una distancia de unos 30 kilómetros (Pozueta (dir.), Lamíquiz \& Porto, 2009), dejaremos este último dato como techo (Cuadros 6a y 6b).

Estos datos corroboran los defendidos en la metodología propuesta, por lo tanto utilizaremos para establecer el tipo de accesibilidad de cada itinerario trazado los expuestos en el Cuadro 2.

\begin{tabular}{rlcccc} 
tipo & $\mathbf{k m}$ recorridos & horas & minutos & tiempo & velocidad promedio $\mathbf{k m} / \mathbf{h}$ \\
bici & 20,61 & 1 & 9 & 1,15 & 16,80 \\
\hline peatón entrenado & 23,38 & 4 & 41 & 4,68 & 5,00 \\
\hline peatón medio & 9,75 & 1 & 57 & 1,95 & 5,00 \\
\hline peatón con nec. esp & 6,83 & 1 & 43 & 1,71 & 4,00
\end{tabular}

Cuadro 6a. Relación entre tiempos (eje x) y distancias (eje y) de los distintos usuarios de las vías de conexión con las áreas naturales. (Fuente: Elaboración propia). 


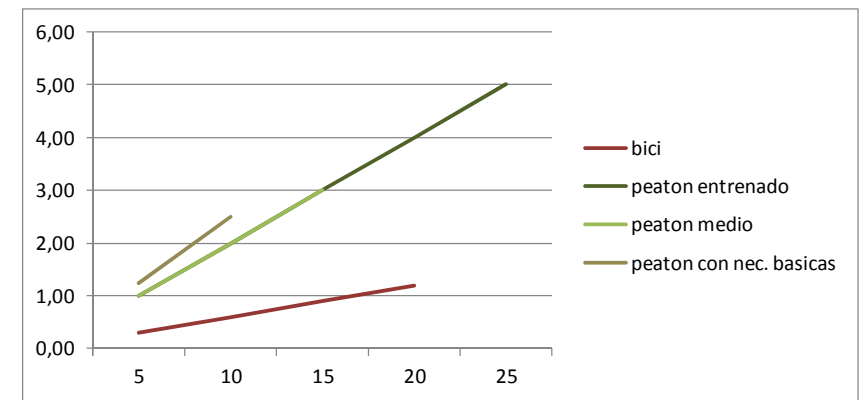

Cuadro 6b. Relación entre tiempos (eje x) y distancias (eje y) de los distintos usuarios de las vías de conexión con las áreas naturales. (Fuente: Elaboración propia).

Según estos criterios, se define la accesibilidad de los distintos puntos elegidos en Madrid a las áreas naturales. Para ello, se determinará en porcentaje cuántas de las conexiones posibles son accesibles según las hipótesis establecidas, es decir, como máximo en una jornada caminando. La accesibilidad se estudia en cuatro niveles según el grado de preparación del peatón.

Se ha aportado un dato adicional al análisis, se ha denominado "factor desvío" y constituye la relación entre la distancia en línea recta al punto de llegada y la distancia efectiva una vez superadas las barreras interpuestas en el camino. A la vista de los planos se concluye que un factor de desvío óptimo equivale a 0,8-0,9 (valores cercanos a 1, obviamente) y los razonables equivalen a 0,7 , constituyendo los caminos más afectados por estos desvíos los que se acerquen a 0,5 .

Conclusiones sobre la accesibilidad de los distritos de Madrid con las áreas naturales periurbanas

El resultado de analizar la accesibilidad desde cada distrito determina una definición de accesibilidades por sectores de la ciudad según los tipos de peatón establecidos, obteniendo los siguientes resultados:

\begin{tabular}{rcccccc}
\hline \begin{tabular}{l} 
ACCESIBILIDAD POR DISTRITO \\
\hline NOMBRE DISTRITO
\end{tabular} & ACCESIBILIDAD BÁSICA & $\begin{array}{c}\text { ACCESIBILIDAD } \\
\text { MEDIA }\end{array}$ & $\begin{array}{c}\text { ACCESIBILIDAD } \\
\text { LIMITADA }\end{array}$ & NO ACCESIBLE & FACTOR DESVÍO \\
\hline I.- Fuencarral el Pardo & $14 \%$ & $14 \%$ & $43 \%$ & $29 \%$ & $\mathbf{0 , 6 9}$ \\
\hline II.- - Barajas & $0 \%$ & $7 \%$ & $64 \%$ & $29 \%$ & $\mathbf{0 , 6 4}$ \\
\hline III.-Villa de Vallecas & $0 \%$ & $7 \%$ & $50 \%$ & $43 \%$ & $\mathbf{0 , 6 5}$ \\
\hline IV.- La Latina & $7 \%$ & $0 \%$ & $64 \%$ & $29 \%$ & $\mathbf{0 , 6 9}$ \\
\hline V.- Chamberí & $0 \%$ & $14 \%$ & $79 \%$ & $7 \%$ & $\mathbf{0 , 7 5}$ \\
\hline
\end{tabular}

Cuadro 7. Accesibilidad por distritos. (Fuente: Elaboración propia).

Así vemos como los sectores que tienen una accesibilidad universal son los Norte, por su cercanía a El Pardo, y el sector Suroeste, por su cercanía a la Casa de Campo, incluida en este estudio. Accesibilidades medias tienen todos los sectores excepto el sector Suroeste que, sin embargo, tenía accesibilidad básica por estar muy cercano a la Casa de Campo.

\section{Carencias de los caminos que conectan las áreas de Naturaleza con Madrid}

Las conexiones de la ciudad con sus áreas naturales se desarrollan, inevitablemente, a través de un territorio fragmentado por múltiples vías de circulación con un uso eminente del vehículo privado. Esto genera desvíos a veces excesivos y prolonga el tiempo de recorrido. 
Los itinerarios que cruzan por el centro de la ciudad a veces tienen que compartir espacio con otros medios de transporte que, en muchas ocasiones, son causas de accidentes. Las secciones necesarias, en algunos casos, no son posibles por el espacio disponible entre edificios o las invasiones de vehículo privado en el espacio público. Puede haber problemas de ruido o contaminación ambiental.

En el extrarradio estos espacios públicos, en ocasiones, están vacíos de significado relevados por las nuevas ágoras de la modernidad y, en consecuencia, degradados (Lamíquiz, 2011). Las carencias relacionadas con estos corresponden a la animación o el atractivo visual y, como consecuencia de una falta de animación, inseguridad ciudadana.

Existe un sinfín de caminos rurales prácticamente imposibles de utilizar porque, por un lado, no están señalizados y, por otro, acaban estrellándose con las infraestructuras de transporte. En ellos habría que resolver el tema del atractivo o animación, la seguridad ciudadana y la protección contra el clima.

Son itinerarios que sobrepasan las distancias sugeridas por varios autores: 500 metros en recorridos peatonales, 7 y 10 kilómetros para recorridos en bicicleta (Pozueta, 2009; Molina \& Sanz, 1996), tienen finalidades distintas a $\operatorname{estos}^{6} \mathrm{y}$, por lo tanto, deben ser concebidos de manera diferenciada.

Existe un débil y escaso apoyo institucional a lo peatonal, aunque incipiente en escalas más locales. En el caso de Madrid, sólo se menciona la necesidad de lo peatonal en el documento Madrid: Movilidad Urbana Sostenible: Un reto energético y ambiental, una guía con buenas intenciones pero que al fin y al cabo será difícil llevar a la práctica si no existe ningún Plan Director de Movilidad Peatonal. ${ }^{7}$

Es destacable, por otro lado, el esfuerzo realizado en estos últimos años por la movilidad en bicicleta, aunque existe una asociación, a veces indebida, con itinerarios peatonales. La coexistencia de estos dos tipos de movilidad, en numerosos casos, es motivo de conflicto.

En conclusión, para que la red de espacio público, articule el entorno natural y la ciudad es necesario:

- Que los itinerarios tengan función, variedad, seguridad y atractivo (Pozueta (dir.), Lamíquiz \& Porto, 2009).

- Que las autoridades tomen conciencia de la necesidad de introducción de Naturaleza en las ciudades (Comisión Europea, 2010a; Fariña, 2009).

\section{Conclusiones y futuras investigaciones}

\section{Conclusiones}

Este trabajo se ha propuesto comprobar la viabilidad de acercar las áreas naturales a las ciudades mediante el método propuesto. Este método se ha verificado mediante la aplicación al caso de Madrid. Se han determinado los itinerarios que, cumpliendo con los requisitos y necesidades de la marcha a pie, tengan una accesibilidad universal y para ello se han categorizado

\footnotetext{
${ }^{6}$ La causa fundamental de que estos caminos tengan sentido es el motivo mismo del viaje. El ocio, el deporte, la salud u otros motivos espirituales o culturales propiciarán el encuentro con la Naturaleza.

${ }^{7}$ Como por ejemplo ocurre en otras ciudades como Vitoria, en su Plan Director de Movilidad Peatonal.
} 
tres tipos de peatones: con necesidades especiales, peatón tipo o medio y peatón entrenado o con condiciones físicas superiores a la media.

El desarrollo de este trabajo ha puesto de manifiesto que las características particulares que tienen estos caminos hacen necesario un estudio específico que contemple los condicionantes de la marcha a pie en función de las características de los distintos entornos recorridos (urbano, periférico y rural). Se han comprobado las diferencias existentes entre la marcha a pie en la ciudad y la que trasciende de los límites de ésta para llegar a las áreas naturales y se han apuntado las características fundamentales que los diferencian (motivación de la marcha a pie, distancias o accesibilidad, diferenciación de secciones, usos asociados, necesidad de señalización, origen y destino).

Un caso considerado aparte es la bici. Para este medio de locomoción, en la definición de accesibilidad interviene el factor velocidad. Es un medio de transporte más eficaz en cuanto a distancias, pero tiene el problema de tener que salvar ciertas barreras a través de pasarelas elevadas, ya que muchas de ellas no cuentan con el ancho y las pendientes adecuadas para la bici. Como ya se citó en este trabajo, se ha incluido este medio de transporte porque puede ser más eficaz en una ciudad de la escala de Madrid. Este medio de transporte puede dar sentido a los itinerarios que, por la accesibilidad de los mismos, el peatón medio y el peatón con necesidades especiales no puedan abarcar.

\section{Líneas de investigación futuras}

La estructura verde de la ciudad, en concreto las teorías sobre green ways y greenbelts, puede tener una relación interesante con el tema estudiado, ya que acerca e introduce la Naturaleza en las ciudades y puede constituir itinerarios ideales a la hora de salir de las ciudades hacia las zonas naturales. Los objetivos de estas estructuras son muy distintos a los planteados y por ello no se han tratado a priori en este trabajo, ya que unos intentan contener el crecimiento de las ciudades y otros conectar áreas verdes para aumentar la biodiversidad.

Una carencia manifiesta de las actuales rutas para salir de las ciudades, y en general de los recorridos peatonales, es una mala señalización, encontrándose en el estudio de caso señalizaciones a base de grafitis y elementos no estandarizados. Éste constituye un punto importantísimo a la hora de la utilización de estos recorridos, como su mantenimiento a lo largo del tiempo y la correcta utilización por parte del usuario.

Uno de los condicionantes de la marcha a pie la constituye la configuración propia de la vía. Un estudio que podría dar continuidad a este trabajo sería el estudio del diseño de las secciones de este tipo de vías.

La práctica totalidad de los itinerarios que dan acceso a las áreas naturales han sido trazados bien por tradición, por historia o por nuevas costumbres difundidas rápidamente gracias a las nuevas tecnologías. El usuario así ha definido sus propias rutas. La cuestión a resolver en este caso sería qué papel puede tener el propio peatón en el diseño de estas vías.

En este trabajo se han apuntado las diferencias de la marcha peatonal urbana y la que supone un desplazamiento hasta las áreas de Naturaleza cercanas. Existen limitaciones en el trabajo a la hora de definir los condicionantes de la marcha a pie en los ámbitos periféricos y rurales. La definición de estos y el contraste con un estudio de caso puede ser una futura línea de investigación. 


\section{Referencias}

\section{Libros y publicaciones}

ALCOLEA MORATILLA, M. Á. (2005). "La función residencial y los problemas ambientales en el distrito de Madrid", Observatorio Medioambiental, $n^{\circ}$ 8, pp. 31-53.

ELKOUSS LUSKI, E. (2006). “La accesibilidad: Hacia la plena integración del discapacitado en el entorno urbano natural”, Cuadernos de Investigación Urbanística, $\mathrm{n}^{\circ} 46$.

EVANS, A. (1997). “Limitaciones al crecimiento urbano, 'cinturones verdes' y economía”, Estudios Públicos, n 67.

FARIÑA TOJO, J. \& NAREDO PÉREZ, J. M. (2010). Libro blanco de la sostenibilidad en el planeamiento urbanístico español. Madrid: Ministerio de Vivienda.

GARCíA PALOMARES, J. C. \& GUTIÉRREZ PUEBLA, J. (2005). "Cambios en la movilidad en el área metropolitana de Madrid: el creciente uso del transporte privado", Anales de Geografía, n² 25, pp. 331-351.

HERNÁNDEZ AJA, A. (1996). "Análisis de estándares de calidad de las ciudades españolas", Cuadernos de Investigación Urbanística, $\mathrm{n}^{\circ} 11$.

HIGUERAS GARCÍA, E. (2009). El reto de la ciudad habitable y sostenible. Pamplona: Editorial DAPP.

JIM, C. \& CHEN, J. (2008). “Ecosystem services and monetary values of urban forests in China”, Cities, $n^{\circ} 26, p p$. 187-194.

JIM, C. (2013). "Sustainable urban greening strategies for compact cities in developing and developed economies", Urban Ecosystems, $\mathrm{n}^{\circ} 16$, pp. 741-761.

JIMÉNEZ JIMÉNEZ, M. (2009). "Sistema de parques: origen y evolución de un principio "estructurador" de lo urbano", Ciudades, $\mathrm{n}^{\circ} 12$.

KABISCH, N. \& HAASE, D. (2013). "Green spaces of European cities revisited for 1960-2006", Landscape and urban planning, $\mathrm{n}^{\circ} 110$, pp. 113-122.

LAMÍQUIZ DAUDÉN, F. J. (2011). Implicaciones de la accesibilidad configuracional en la movilidad peatonal. El caso de Madrid. Tesis Doctoral. Escuela Técnica Superior de Arquitectura. Universidad Politécnica de Madrid.

FADIGAS, L. (2009). “La estructura verde en el proceso de planificación urbana”, Ciudades, n 12, pp. 33-47.

MENDOZA-HAUCHECORNE, E. (2013). "La movilidad urbana como modus operandi que edifica las ciudades", Economía, Sociedad y Territorio, vol. XIII, $\mathrm{n}^{\circ} 42$.

MOYA GONZÁLEZ, L. (2006). "Estudio de medidas de movilidad ambiental en el entorno europeo", Cuadernos de Investigación Urbanística, $\mathrm{n}^{\circ} 49$.

MUNTON, R. (1983) London's green belt: containment in practice. Londres: Allen and Unwin.

POZUETA, J. (dir.); LAMÍQUIZ, F. J. \& PORTO, M. (2009). La ciudad paseable. Recomendaciones para la consideración de los peatones en el planeamiento, el diseño urbano y la arquitectura. Madrid: CEDEX, Ministerio de Fomento y Ministerio de Medio Ambiente y Medio Rural y Marino.

RUEDA PALENZUELA, S. (2011). “El urbanismo ecológico”, TRIA: Rivista Internazionale di Cultura Urbanistica, nº 6.

TAYLOR LOVELL, S. \& TAYLOR, J. R. (2013). “Supplying urban ecosystem services through multifunctional green infrastructure in the United States", Landscape Ecology in Review, n 28, pp. 1.447-1.463.

DE TERÁN TROYANO, F. (1970). “Actualidad de las nuevas ciudades de Gran Bretaña”, Ciudad y Territorio: Revista de Ciencia Urbana, $n^{\circ} 4$.

TOBIAS, S. (2012). “Preserving Ecosystem Services in Urban Regions: Challenges for Planning and Best Practice Examples from Switzerland", Integrated Environmental Assessment and Management, vol. 9, n 2, pp. 243-251. 
TODARO, V. (2007). “Procesos de integración entre redes ecológicas e instrumentos de planificación”, Cuadernos de Investigación Urbanística, $\mathrm{n}^{\circ} 54$.

WILLIAMS, R. (2001). El campo y la Ciudad. Buenos Aires: Paidó.

\section{Publicaciones institucionales}

GENERALITAT DE CATALUNYA. (2008). Pla de Mobilitat Urbana. Avaluació ambiental en la planificació de la mobilitat. Departament de Medi Ambient.

AYUNTAMIENTO DE BACELONA. (2008). Plan de Movilidad (PMU) 2013-2018.

AYUNTAMIENTO DE MADRID (2007). Plan Director de Movilidad Ciclista.

- (2010). Movilidad Urbana Sostenible: Un reto energético y ambiental.

- (2014a). Plan General de Ordenación Urbana.

- (2014b). Plan de Movilidad Madrid.

AYUNTAMIENTO DE MÁLAGA. (2011). Avance Plan de Movilidad.

AYUNTAMIENTO DE PAMPLONA. (2007). Plan de Movilidad Urbana Sostenible de la Comarca de Pamplona.

- (2010). Pacto local de movilidad sostenible de Pamplona.

AYUNTAMIENTO DE SEVILLA. (2006). Plan de Movilidad del área de Sevilla.

AYUNTAMIENTO DE VITORIA-GASTEIZ. (2006a). Informe de Movilidad y Sostenibilidad.

- (2006b). Memoria de avance 2007.

- (2006c). Plan de Movilidad Sostenible y de Espacio Público.

- (2010). Plan director de Movilidad Ciclista 2010-2015.

COMUNIDAD AUTÓNOMA DE ANDALUCÍA. (2013) Revisión del Plan de Infraestructuras para la Sostenibilidad del Transporte en Andalucía.

- (2014). Plan Andaluz de la Bicicleta. PAB 2014-2020.

COMISIÓN DE LAS COMUNIDADES EUROPEAS. (2010). Plan de acción de movilidad europeo.

COMISIÓN EUROPEA. (1990). Libro verde sobre el medio ambiente urbano. Comunicación de la Comisión al Consejo y al Parlamento. Bruselas.

- (2001). COST Action C6. A city for pedestrians: Policy-making and implementation. Dominique Fleury Town and infrastructure planning for safety and urban quality for pedestrians.

- (2003). Seguridad y reducción de accidentes. Programa Marco I+D+i n n 4.

- (2005a) Cost Action C11. Cap. 1: “Greenstructure and Urban Planning”. COST Office.

- (2005b) Cost Action C11. Cap. 2: “Cases”. COST Office.

- (2005c) Cost Action C11. Cap. 3: “An ecological approach to green structure planning”. COST Office.

- (2005d) Cost Action C11. Cap. 4: "Human issues". COST Office.

- (2005e) Cost Action C11. Cap. 6: "Epilog (Issues of the final open seminar in Ceske Budejovice)". COST Office

- (2005f). New Means to promote Pedestrian Traffic in Cities. Programa PROMPT.

- (2006). Cost Action 358. Pedestrians' quality needs. 165th CSO meeting, 27-28 June. 
- (2010a). Estrategia para una movilidad sostenible de los desplazamientos en Europa. Project E-CoSMOS.

- (2010b) Comité Económico y Social Europeo. Plan de acción sobre la movilidad urbana. COM(2009) 490 final.

INTERNATIONAL CHARTER FOR WALKING. (2007). Carta internacional del caminar.

Disponible en: http://www.Walk21.com/charter/documents/Carta_Internacional_espanol_03-07.pdf.

MINISTERIO DE MEDIO AMBIENTE. (2001). Sistema español de indicadores ambientales: área de medio urbano. Dirección General de Calidad y Evaluación Ambiental.

MINISTERIO DE FOMENTO Y MINISTERIO DE MEDIO AMBIENTE Y MEDIO RURAL Y MARINO. (2009). La estrategia española de movilidad sostenible 2009.

MINISTERIO DE FOMENTO. (2005). Plan estratégico de infraestructuras y transporte (PEIT) 2005-2020.

\section{Referencias en la web}

COMUNIDAD DE MADRID. (2014a). Nomecalles.

Disponible en: http://www.madrid.org/nomecalles.

- (2014b) Proyecto Sistema de Información sobre Ocupación del Suelo de España (SIOSE).

Disponible en: http://www.madrid.org/cartografia/visorSiose/html/visor.htm.

- (2014c): Instituto de Estadística de la Comunidad de Madrid.

Disponible en: http://www.madrid.org/iestadis/.

CORINE LAND COVER (CLC). (2006).

Disponible en: http://www.ign.es/ign/layoutln/corineLandCover.do.

MINISTERIO DE AGRIGULTURA, ALIMENTACIÓN Y MEDIO AMBIENTE. (2014). Visor del Fega, SigPAC.

Disponible en: http://sigpac.mapa.es/fega/visor/.

MINISTERIO DE FOMENTO. (2014a) Instituto Geográfico Nacional.

Disponible en: http://www.ign.es/iberpix2/visor/

- (2014b). Planea. Dirección General de Urbanismo y Estrategia Territorial.

Disponible en: http://www.madrid.org/cartografia/planea/index.htm.

- (2014c) Sistema de Información Urbana (SIU).

Disponible en:

http://www.fomento.gob.es/MFOM/LANG_CASTELLANO/DIRECCIONES_GENERALES/ARQ_VIVIENDA/SUELO_Y_POLI TICAS/SIU/. 